\title{
Follicle-stimulating isohormones: regulation and biological significance
}

\author{
V. Padmanabhan, J. S. Lee and I. Z. Beitins* \\ Department of Pediatrics, Reproductive Sciences Program, The University of Michigan, \\ Ann Arbor, MI 48109, USA
}

\begin{abstract}
Follicle-stimulating hormone (FSH) is a key hormone in the regulation of follicular development. Although the existence of FSH heterogeneity is well established, the physiological significance of this pleomorphism remains unknown. Observed changes in circulating FSH heterogeneity during critical reproductive events such as puberty and reproductive cyclicity suggest that different combinations of FSH isoforms reach the target sites during different physiological states to influence a variety of biological end points such as cellular growth, development, steroidogenesis and protein synthesis. Considering that these FSH isoforms have different physicochemical properties and potential to bind not only their cognate receptors but also structurally related, non-FSH receptors with various affinities, the regulatory implications of FSH heterogeneity in modulating the various FSH-induced functions are enormous. However, assigning functional significance to FSH heterogeneity has been hampered because of (1) difficulties associated with procurement of highly purified, naturally occurring, circulating FSH isoforms; (2) absence of reference standards that contain the entire repertoire of FSH isoforms present in biological fluids; and (3) specificity issues inherent to the detection systems used. If particular FSH isoforms do possess selective biological functions, specific combinations of FSH isoforms could be generated to regulate fertility in farm animals and humans.
\end{abstract}

\section{Introduction}

Although the existence of protein heterogeneity is now uncontested, the intracellular mechanisms governing protein synthesis, post-translational modifications and post-secretion fates are poorly understood. Proteins emerging from the ribosome have a great variety of potential fates, depending on the presence or absence of critical amino acid sequences. These amino acid sequences constitute target signals that determine spatial configuration, folding and post-translational modifications (including the addition and trimming of carbohydrate units, stability, packaging in granules and secretion). Structural features (such as tertiary configuration, carbohydrate composition) determine the metabolic fate of the protein in the peripheral circulation. Although the structure of the oligosaccharide chains of glycoproteins is an important determinant of the circulating half-life, protein structure and exposed epitopes are important for target cell receptor binding and signal transduction. For these and other reasons, it appears that the resulting mix of heterogeneous hormonal isoforms exert effects at the target site that cannot be interpreted properly by conventional radioimmunoassays alone.

\section{Existence, Origin and Functional Attributes of FSH Isoforms}

\section{Existence of FSH isoforms}

Use of various separation techniques, such as chromatofocusing, isoelectric focusing and gel electrophoresis, has unequivocally established that FSH occurs in multiple forms both in the

- Current address: National Center for Research Resources, National Institute of Health, Bethesda, MD. 
pituitary and circulation (reviewed in Chappel et al., 1983; Beitins and Padmanabhan, 1991; UlloaAguirre et al., 1995). The more discriminating the separation technique the more complex the distribution profiles of FSH isoforms. For instance, Burgon et al. (1993), using isoelectric focusing and high performance anion exchange chromatography, identified more than 20 human pituitary isoforms. A complete array of FSH isoforms ranging from basic to strongly acidic pI values is found both in the pituitary and circulation (reviewed in Chappel et al., 1983; Beitins and Padmanabhan, 1991; Ulloa-Aguirre et at., 1995). In some physiological and pharmacological conditions, the distribution of circulating and pituitary FSH isoforms appears to be similar, while in others they differ.

\section{Origin of FSH heterogeneity}

There are several potential sites at which changes in FSH heterogeneity can originate. At the pituitary, the array of FSH isoforms that exist during different physiological states may represent merely FSH at different stages in the biosynthetic pathway. Alternatively, changes in FSH heterogeneity may be the outcome of alterations in post-translational processing and a function of glycosylation changes. In this context, it is of interest that the activities of glycosyltransferases and sialotransferases, enzymes involved in sulphation and sialylation of luteinizing hormone (LH) and FSH, vary with the physiological state in the pituitary (Dharmesh and Baenziger, 1993; DamianMatsumura et al., 1998). This may contribute to potential differences in glycosylation of pituitary gonadotrophins.

At the circulatory level changes in FSH heterogeneity may originate from regulated preferential secretion, peripheral modification, or differences in metabolic clearance. In vitro studies (reviewed in Chappel et al., 1983; Ulloa Aguirre et al., 1995) and recent in vivo studies in sheep (Lee et al., 1998) suggest that the pituitary has the ability to secrete different combinations of FSH isoforms depending on the physiological status of the animal.

\section{Functional attributes of FSH isoforms}

The nature of the oligosaccharide side chains varies considerably and may be manifested as changes in carbohydrate content, length and branching of the side chain and the associated charge. These structural differences appear to dictate the receptor binding ability, in vitro and in vivo biological activities, and circulatory half-lives of the FSH isoforms. Most of the earlier work that used biological to immunological ratios to estimate FSH biopotencies concluded that in vitro biological activity of the FSH isoform is positively correlated with the pI value of the isohormone; the FSH isoforms with higher $\mathrm{pI}$ values have higher in vitro biological activities than those with lower pI values (reviewed in Chappel et al., 1983; Beitins and Padmanabhan, 1991; Ulloa-Aguirre et al. 1995). An exception to this rule is that the basic FSH isoforms that elute in the void volume of the chromatofocusing column ( $\mathrm{pH}$ gradient 7.4-4.0) appear to act as antagonists of FSH (Dahl et al., 1988; Timossi et al., 1998).

The assumption in the use of B.I ratios for estimating FSH biopotency has been that the immunopotencies are similar between different FSH isoforms. More recent studies that used highly purified FSH isoforms of defined mass (not adjusted for carbohydrate content and oxidative losses) have raised questions about this assumption and shown the contrary, namely that FSH immunopotencies of different FSH isoforms vary (Burgon et al., 1993; Stanton et al., 1996). It then follows that earlier biopotency estimates that used immunoassays to determine gonadotrophin protein mass may be inaccurate. On the basis of the high correlation between immuno-, radioreceptor and in vitro bioactivity of 15 purified human FSH isoforms ( $>90 \%$ purity) in the pI value range of 3.63-5.13, Burgon et al. (1993) concluded that: (1) current immunoassays are measuring mass in combination with some measure of bioactivity and (2) an immunoassay directed to the 'invariant region of the molecule' not affected by glycosylation differences is required to assess the true bioactivity of the FSH isoform. Interestingly, in spite of the high correlation between the 
immuno-, radio-receptor and in vitro bioactivities of individual isoforms, these investigators also noted significant differences in ratios of activities between in vitro bioassays and other methods (Burgon et al., 1993). For example, the in vitro bio-to radio-receptor assay ratio of the human FSH isoform with a $\mathrm{PI}$ value of 4.23 was four times greater than those with pI values of $3.63,3.88,4.07$, 4.85 , and 5.13. Isoforms with higher $\mathrm{pI}$ values, such as those reported to have antagonistic properties (Dahl et al., 1988; Timossi et al., 1998) or the less acidic FSH isoforms (pI > 5.4) seen during puberty (Padmanabhan et al., 1992), have not been evaluated. Differences in assay ratios from the carefully characterized study of Burgon et al. (1993) and the 5-8-fold differences in immuno-, radio-receptor and in vitro bioactivities of the various human $\mathrm{FSH}$ isoforms indicate that structural heterogeneity of FSH does contribute to functional differences.

In terms of the circulatory half-life, many studies using chemically or enzymatically modified FSH have shown that the less acidic FSH isoforms have shorter half-lives than the more acidic FSH isoforms (reviewed in Chappel et al., 1983; Beitins and Padmanabhan, 1991; Ulloa-Aguirre et al., 1995). Differences in half-life in these instances appear to relate to the degree of sialylation (Morell $e t$ al., 1971). Although most studies follow this general rule, others have failed to observe such relationships (Robertson et al., 1991). Assuming that the FSH isohormones with shorter circulatory half-lives have higher biological activity in vitro, the issue is to determine whether measures of in vitro biological activity are meaningful in interpreting consequences in vivo. Studies by Stanton et al. (1996) show a 16-fold range in in vivo activities between various LH isoforms. These changes parallel estimates of bioactivity in vitro. This finding suggests that differences in bioactivity in vitro and not the large differences in circulatory half-life are the key determinants of bioactivity in vivo as assessed in short-term assays (long-term consequences were not assessed).

The effector mechanisms by which FSH elicits its target cell action appear also to be conducive for rapid functional interactions between FSH and its receptor. For example, minutes after addition, FSH elicits increases in intracellular calcium (Flores et al., 1990). Furthermore, effective activation of adenylate cyclase appears possible under conditions of intermittent receptor activation (Spiegel et al., 1992). In this context it is of interest that FSH is secreted both in a basal and pulsatile mode (Padmanabhan et al., 1997a). Some evidence also indicates that what is secreted in pulses is of different molecular nature than that secreted in the basal mode (reviewed in Ulloa-Aguirre et al., 1995). It still remains to be addressed whether the pulsatile inputs are perceived by the target site as such, and if so, do they respond differently to this intermittent activation as opposed to FSH secreted in the basal mode.

Although many issues still remain to be addressed, it is clear that a combination of circulating gonadotrophin isoforms reaches target tissues and influences a variety of biological end points such as cellular growth, development, steroidogenesis and synthesis of proteins. FSH isoforms possessing prolonged circulation times may have the potential to provide a long-acting stimulus for the progression of maturational events. In contrast, isoforms with shorter half-lives that are secreted intermittently may provide an acute yet potent stimulus (Chappel et al., 1983; Beitins and Padmanabhan, 1991). Relative proportions of the various gonadotrophin isoforms within the circulation, therefore, have the potential to exert qualitatively different effects on target tissues.

\section{Methodological Considerations in Assessing FSH Heterogeneity}

\section{Utility of $B: I$ ratios}

Initial studies of FSH heterogeneity relied heavily upon comparative bioactivity (B) and immunoreactivity (I) estimates. In these instances changes in B:I ratios were taken to imply that there was a change in the constituent mix of FSH isoforms. More recent studies have questioned this concept (Jaakkola et al, 1990, Simoni et al., 1994). Conclusions on hormonal heterogeneity derived from studies of B:I ratios of FSH must be drawn with caution and may suffer from two major drawbacks. First, it needs to be proven that the differences in measured bioactive FSH and immunoreactive FSH are not simply the result of differences in the effects of interfering substances 
on the measurement system but result from an ability to discriminate among isoforms. For example, RIAs using different combinations of standards and antisera (with different epitopic recognition) can differentially recognize gonadotrophin mixtures (Simoni et al., 1994). Bioassays suffer in that substances present in the test material can interfere (Simoni et al., 1991) or modulate the effectiveness of FSH (reviewed in Beitins and Padmanabhan, 1991; Chappel, 1995) leading to erroneous estimates.

Second, the FSH standards used in various immuno- and bioassays add another source of error when estimating changes in FSH B:I ratios. The distribution patterns of FSH isoforms of pituitary and urinary FSH standards appear to have sparser representation of less acidic FSH isoforms than those found in circulation and unpurified pituitary extracts (Simoni et al., 1993a, 1993b). This finding suggests that the standards that are currently in use in the various assays do not have the full array of FSH isoforms that are present in biological fluids. Although it is ideal to have a reference preparation that contains the repertoire of FSH isoforms present in the biological fluid being measured, this does not appear practical in view of the high variability associated with its composition during different physiological states. At the very minimum, documentation of changes in FSH heterogeneity using FSH B I ratios requires careful characterization of the assay systems and use of the same reference preparations. Unfortunately, many of the earlier studies reporting changes in FSH B:I ratios used different FSH standards to estimate the immuno- and biopotencies. In fact, the first study that challenged the utility of B:I ratios itself used two different standards in their immuno- and bioassays (Jakkola et al., 1990). The specificity of the monoclonal antibodies also adds an additional problem in that they fail to recognize all variants (Pettersson and Soderholm, 1991).

In terms of bioassays, the biopotency estimate of the FSH isoform may also vary with the biological end point or assay system chosen. When CAMP is used as the endpoint in the Sertoli cell bioassay, deglycosylated ovine FSH appears to be inactive (Fig. 1) (Padmanabhan, 1995). On the contrary, when oestradiol is chosen as the end point, deglycosylated FSH stimulates oestradiol production. Species specificity of the bioassay is also a consideration. For instance, Ding et al, (1991) have shown that the bioactive LH estimates and the B:I ratios vary markedly depending on whether the mouse Leydig cell, rat interstitial cell or human granulosa cell bioassay is used to measure LH bioactivity. Bioassays of FSH that use cells transfected with recombinant FSH receptors (Gudermann et al., 1994; Christine-Maitre et al., 1996) and CAMP as the end point also pose a problem because they do not measure a biological end point and ignore involvement of other second messenger systems involved in FSH signal transduction.

Taking all aforementioned caveats into consideration, it appears that differences in FSH B:I ratios reported by different investigators during similar physiological situations are a direct function of the assay, the standard and end point used. For instance, FSH B:I ratios are constant throughout the cycle (Jia et al., 1986), highest during the preovulatory period (Padmanabhan et al., 1988a; Wide and Bakos, 1993; Zambrano et al., 1995), or maximal in the luteal phase (Christine-Maitre et al., 1996) of the human menstrual cycle. Therefore, when comparing biological potency estimates, it is important to consider the species, the cellular types, as well as the relative endpoints of the bioassay used by the various investigators. Assuming that assay systems are controlled carefully, changes in $B$ :I ratio at best will point to possible changes in heterogeneity but will require confirmation with other chromatographic approaches.

\section{Chromatographic separation}

Several fractionation approaches have been used effectively to characterize changes in FSH heterogeneity and have been reviewed extensively (Ulloa-Aguirre et al., 1995). Although a broader classification of FSH isoforms can be achieved by gel permeation chromatography, higher resolution has been achieved with techniques that separate on the basis of charge. Some charge-based techniques that have been used extensively are isoelectric focusing, chromatofocusing and analytical zone electrophoresis. These approaches while providing a general assessment of changes, do not separate isoforms to purity which requires multiple chromatographic steps (Burgon et al., 1993). An inherent caveat in these approaches is that estimates of FSH isoform distribution after fractionation are based on immunoassay systems that have their inherent problems. Recent studies of Simoni et al. 

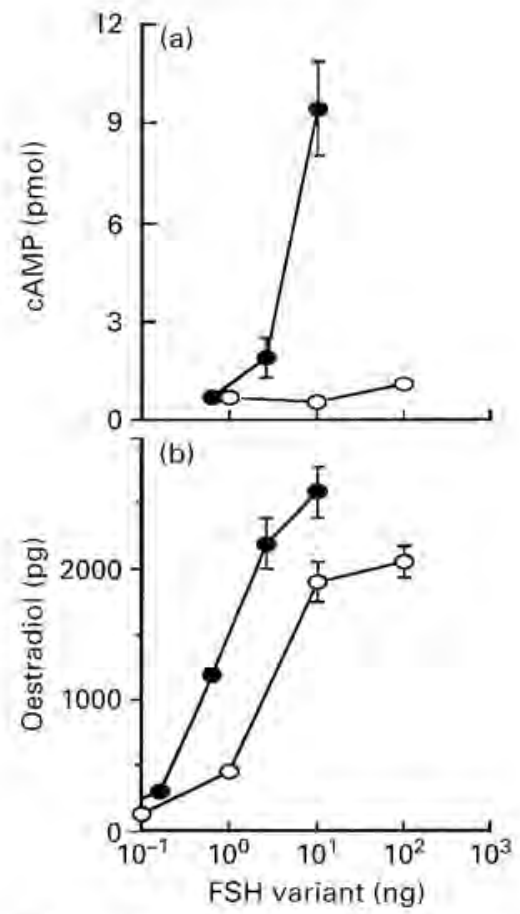

Fig. 1. (a) CAMP, and (b) oestradiol responses of immature rat Sertoli cells to increasing concentrations of native (oFSH) (-) or deglycosylated oFSH (DGoFSH) (O). Sertoli cells from 7-10-dayold rats were cultured using previously validated methods (Padmanabhan et al, 1987). Cell monolayers were exposed to increasing concentrations of oFSH or DG-oFSH for $24 \mathrm{~h}$. Thirty minutes after addition of $\mathrm{FSH}$, a $200 \mu \mathrm{l}$ aliquot of medium was removed from each well for CAMP determinations. Medium concentrations of oestradiol (24 h incubates) and CAMP (30 min incubates) were measured using previously validated assays. Note the differences in cAMP and oestradiol responses to the native and deglycosylated FSH (modified from Padmanabhan et al, 1993).

(1994) have shown that the distribution profiles of FSH after chromatofocusing vary considerably between immunoassays because each assay recognizes individual isoforms of FSH differently.

\section{Regulation of FSH Heterogeneity}

A central theme emerging from investigations of FSH heterogeneity is that the endocrine status regulates the proportion of various FSH isoforms and that these isoforms show distinct actions. 
Although the factors affecting the final distribution of gonadotrophin isoforms within the circulation are multifaceted and complex, it is clear that endocrine changes regulate the proportions of FSH isoforms both within the pituitary and in the peripheral circulation.

\section{Pituitary FSH heterogeneity}

Endocrine regulation of pituitary FSH microheterogeneity has been studied widely in numerous species. Several reviews have addressed the regulation of pituitary heterogeneity in great detail (reviewed in Chappel et al., 1983; Beitins and Padmanabhan, 1991; Ulloa-Aguirre et al., 1995). Qualitative differences in pituitary FSH content have been correlated with age, sex and stage of the oestrous cycle in several species by using various techniques (Blum and Gupta, 1980; Chappel et al., 1983; Ulloa-Aguirre et al., 1995). In general, pituitary FSH is less acidic in female than male pituitaries and young than old animals. Changes in pituitary FSH heterogeneity (predominance of less acidic FSH isoforms) have also been found during onset of puberty in rats (Chappel et al., 1983), but not in heifers (Stumpf et al., 1992).

\section{Circulating FSH heterogeneity}

Regulation of circulating FSH heterogeneity has not received such intense investigation as pituitary heterogeneity, in part due to limitations imposed by relatively low concentrations of circulating FSH. Development of sensitive, in vitro FSH bioassays which use oestradiol as the end point (granulosa cell bioassay: Jia et al., 1986; Sertoli cell bioassay: Padmanabhan et al., 1987) have allowed characterization of changes in circulating bioactive FSH during different physiological states (reviewed in Beitins and Padmanabhan, 1991). Because of the caveats discussed above in using B:I ratios as an index of changes in FSH heterogeneity, in this report, only those studies that used chromatographic approaches either alone or in conjunction with bioactivity measures to assess changes in FSH heterogeneity will be considered. In general, increases in oestradiol and GnRH in gonad-intact models lead tô increases in less acidic FSH isoforms and corresponding increases ín bioactive FSH in the circulation (Fig. 2) (reviewed in Beitins and Padmanabhan, 1991; Ulloa-Aguirre et al., 1995). Administration of GnRH to prepubertal boys (Phillips and Wide, 1994), men (Simoni et al., 1996), and women (Zambrano et al., 1995) increases the release of less acidic FSH isoforms. Treatment of women with GnRIA antagonist, on the other hand, leads to an increase in the circulation of a highly basic FSH isoform (Dahl et al., 1988). This basic FSH isoform was shown to antagonize the action of FSH.

Few studies have also addressed the role of oestradiol in modulating circulating FSH heterogeneity. Studies in humans documenting changes in FSH heterogeneity (shift to a less acidic side) following treatment of postmenopausal women (Wide and Naessén, 1994) or a Turner's girl (Padmanabhan et al., 1988a) with oestrogens provide corroborative evidence in support of a role for oestradiol in modulating circulating FSH heterogeneity. In these instances, since oestrogens can induce their effects via mediation of hypothalamic GnRH secretion, it is difficult to separate the direct effects of oestradiol from mediation via alterations in GnRH input. Studies using ovariectomized, nutritionally growth-retarded (hypogonadotrophic) sheep have shown that pulsatile GnRH administration alone, in the absence of oestradiol, increases LH but does not alter the distribution profile of FSH or bioactive FSH secretion (Fig. 3) (Hassing et al., 1993). In contrast, oestradiol administration to nutritionally growth-retarded lambs leads to an increase in less acidic FSH isoforms (Padmanabhan et al, 1997b) (Fig. 4). Therefore, these findings support a role for oestradiol in mediating FSH heterogeneity.

Progesterone and androgens appear to have an opposite effect to that of oestradiol since they appear to increase the presence of more acidic FSH isoforms (Fig. 2) (reviewed in Ulloa-Aguirre et al., 1995). In the presence of high progesterone, oestradiol fails to increase the presence of less acidic FSH isoforms in the circulation (Wide et al., 1996a). Furthermore, the predominant circulating form of FSH is acidic during the luteal phase of the human menstrual cycle (Padmanabhan et al., 1988a) and 
Neuroendocrine regulation

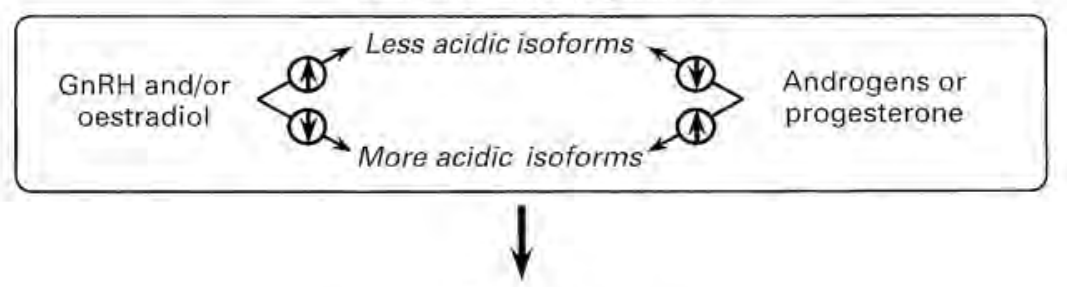

Circulation

\section{Postmenopausal women treated with $\mathrm{GnRH}$ antagonist \\ (1) Basic (antagonistic) isoforms}

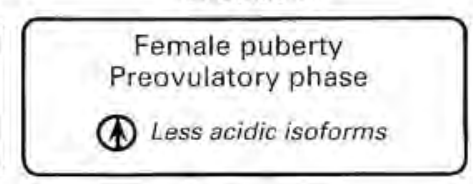

Male puberty and adulthood Male and female senescence Early follicuiar and luteal phase

(1) More acidic isoforms

Fig. 2. Schematic diagram summarizing the neuroendocrine regulation of FSH heterogeneity and the nature of circulating FSH isoforms during different physiological states (reviewed in Beitins and Padmanabhan, 1991; Ulloa-Aguirre et al., 1995). GnRH (particularly in ovary-intact models) and oestradiol appear to increase the proportion of less acidic FSH isoforms and decrease the presence of more acidic FSH isoforms. In contrast, androgens and progesterone appear to increase the concentration of more acidic FSH isoforms. The distribution of FSH isoforms also varies with the physiological state. For example, during the onset of puberty in females and the preovulatory phase greater proportions of less acidic FSH isoforms circulate. In contrast, circulating FSH in males, during female senescence, and the early follicular and luteal phases of the reproductive cycle appear predominantly acidic in nature. Interestingly, treatment of post-menopausal women with a GnRH antagonist increases the release of basic FSH isoforms with antagonistic properties (Dahl et at., 1988).

the prepartum period in cattle (Crowe et al., 1998), when concentrations of progesterone and oestradiol are both high.

In all these studies it is difficult to ascertain whether an increase in less acidic FSH isoforms such as those induced by GnRH or oestradiol is due to selective secretion or metabolic alteration. Studies of Harsch et al. (1993) suggest that metabolic deglycosylation can occur in circulation. More recent studies characterizing FSH distribution near the site of secretion show that oestradiol selectively increases the secretion of less acidic FSH isoforms (Lee et al., 1998). Evidence is also accumulating to show that oestradiol alters the activity of pituitary glycosyl- and sialotransferases (Dharmesh and Baenziger, 1993; Damian-Matsumura et al., 1998), thereby contributing to glycosylation differences. Overall changes in circulating FSH heterogeneity appear to be the sum effect of secretory changes, metabolic alterations and metabolic clearance.

\section{Biological Significance}

When assessing biological significance, it is not sufficient only to show FSH heterogeneity is present and is regulated. It is essential to assess whether such changes are biologically meaningful and to determine whether the naturally occurring FSH isoforms are different functionally. In general, increased release of less acidic FSH isoforms occurs at the onset of puberty and the preovulatory period (Fig. 2). In contrast, more acidic FSH isoforms predominate in males, during senescence in both males and females, and during the early follicular and luteal phases of the oestrous or menstrual cycles (Fig. 2) (reviewed in Beitins and Padmanabhan, 1991; Ulloa-Aguirre et al., 1995).

\section{Changes in FSH heterogeneity may be important in the pubertal process}

Experimental induction of puberty in female lambs increases the release of circulating bioactive FSH in pubertal lambs as compared with prepubertal lambs; this increase is not evident when 

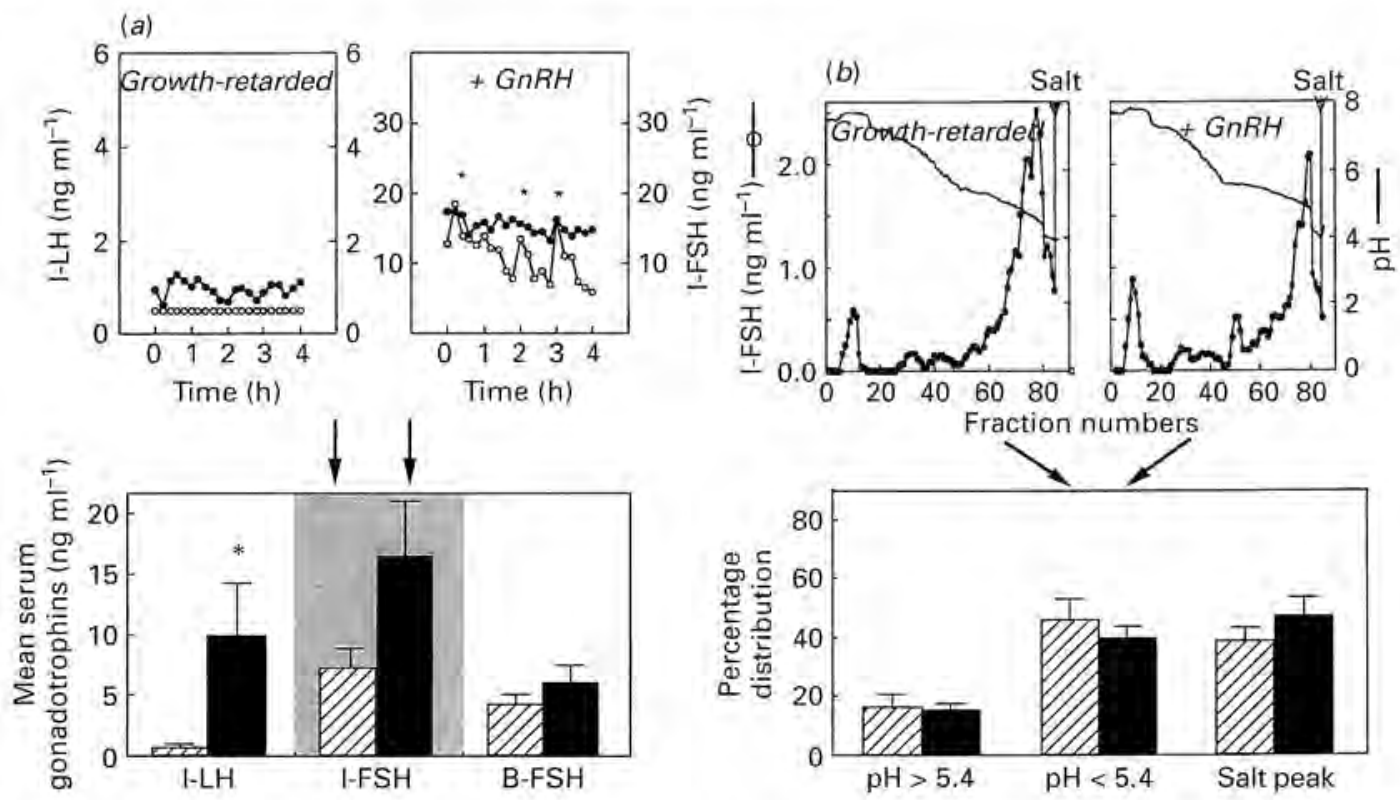

Fig. 3. Changes in circulating concentrations of gonadotrophins and FSH heterogeneity after pulsatile administration of GnRH to ovariectomized nutritionally growth-retarded (hypogonadotrophic lamb) (Hassing et al., 1993). (a) Circulating patterns of immunoreactive (I) FSH in a growth-retarded and GnRH-treated lamb (top) and mean concentrations of I-FSH and bioactive FSH (B-FSH) measured by the Sertoli cell aromatase bioassay (bottom). Circulating patterns and mean concentrations of $\mathrm{I}-\mathrm{LH}$ are provided for comparison. (b) Distribution pattern of immunoreactive FSH (closed circles) after separation of serum from a representative growth-retarded (left) and GnRH-treated (right) lamb by chromatofocusing (top). Percentage distribution of circulating FSH isoforms that eluted at $\mathrm{pH}$ above 5.4, below $\mathrm{pH} 5.4$ and the components bound at the lower limiting pH are shown in the bottom. Values represent mean $\pm \operatorname{SEM}(n=4$ for each group).

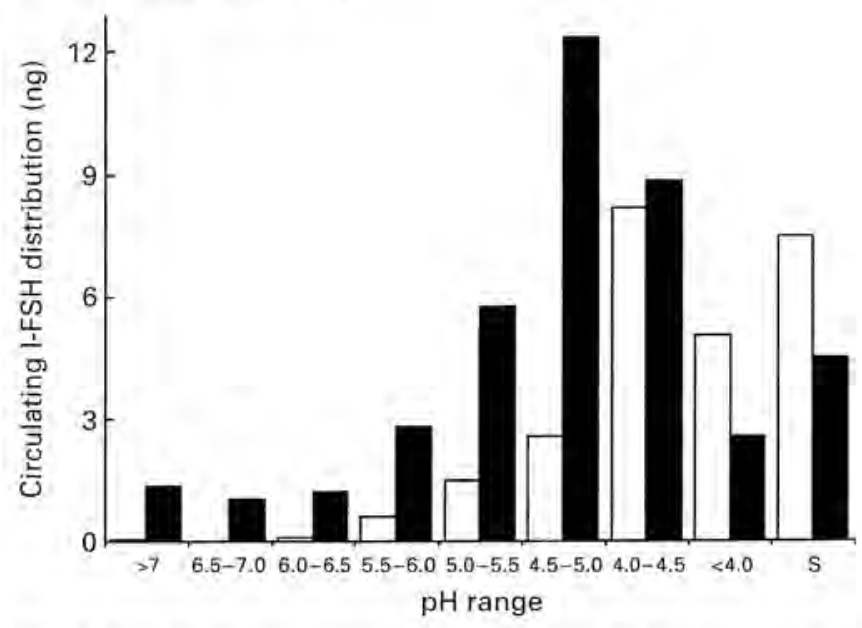

Fig. 4. Distribution pattern of immunoreactive FSH after separation of serum from a representative ovariectomized hypogonadotrophic

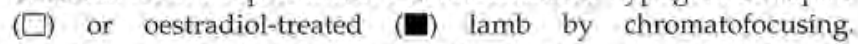
Ovariectomized hypogonadotrophic lambs were treated with follicular phase concentrations of oestradiol (via implants for $18 \mathrm{~h}$ ). Note the shift to the less acidic side of FSH distribution in the oestradiol-treated lamb (Padmanabhan et al., 1997b). 

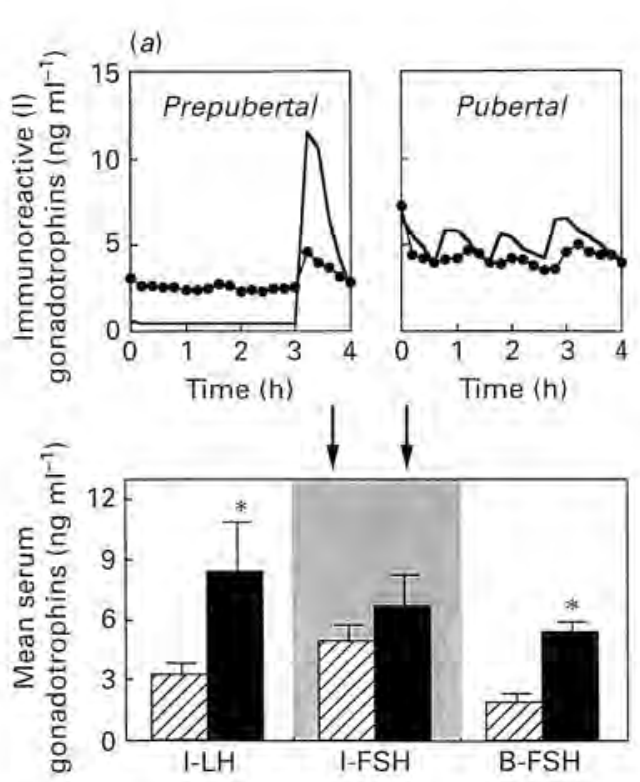
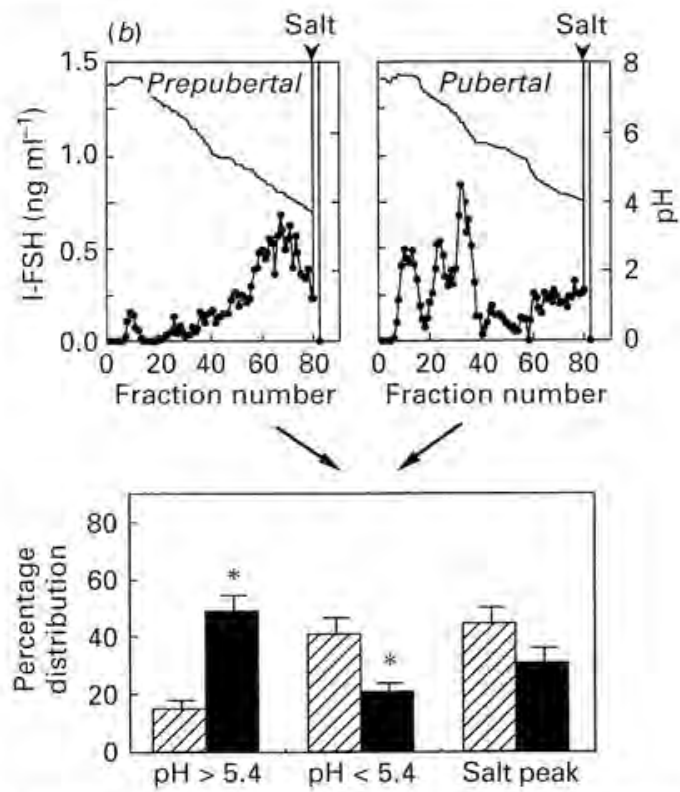

Fig. 5. Changes in circulating concentrations of gonadotrophins and FSH heterogeneity during experimental induction of puberty in female lambs (Padmanabhan et al., 1992). (a) Shown are circulating patterns of immunoreactive (I) FSH in a prepubertal and pubertal lamb (top) and mean concentrations of I-FSH and bioactive FSH (B-FSH) measured by the Sertoli cell aromatase bioassay (SAB) and granulosal cell aromatase bioassay (GAB) in the prepubertal and pubertal lambs (bottom). Circulating patterns and mean concentrations of I-LH are also provided for comparison. Note the selective increase in B-FSH as measured by the SAB and GAB assays at the onset of puberty. (b) Distribution pattern of I-FSH (closed circles) after separation of serum from a representative prepubertal (left) and pubertal (right) lamb by chromatofocusing (top). Note the elution of greater amounts of immunoreactive FSH isoforms in the less acidic region in the pubertal lamb. Percentage distribution of circulating FSH isoforms that eluted at $\mathrm{pH}$ above 5.4 , below $\mathrm{pH}$ 5.4 and the components bound at the lower limiting $\mathrm{pH}$ are shown in the bottom. Values represent mean \pm $\operatorname{SEM}(n=4$ for each group).

measured by a radioimmunoassay (Fig. 5) (Padmanabhan et al., 1992). The increased release of bioactive FSH in pubertal lambs is evident whether measured by the Sertoli or the granulosa cell bioassays. However, quantitative differences in bioactive FSH estimates are evident depending on the bioassay used (reviewed in Ulloa-Aguirre et al., 1995). The increased release of B-FSH in pubertal lambs also is accompanied by a change in the distribution pattern of circulating FSH isoforms (Fig. 5) with increased release of less acidic ( $\mathrm{pI}>5.4$ ) serum FSH isoforms. Considering that pubertal onset is associated with increased gonadal activity, the less acidic FSH isoforms in conjunction with increased secretion of bioactive FSH indicate that the less acidic isoforms may be biologically meaningful and have the potential to provide a potent and acute signal to the ovary. Such changes may be the consequence of increased oestradiol secretion from the ovary.

\section{Changes in FSH heterogeneity may be important in the ounlatory process}

Detailed chromatographic studies characterizing the changes in circulating FSH heterogeneity during the ovulatory cycles have been addressed in humans (Padmanabhan et al, 1988a; Wide and Bakos, 1993; Zambrano et al., 1995) and cattle (Cooke et al., 1997). These studies reveal that increases in less acidic circulating FSH isoforms occur during the preovulatory period. Studies using concanavalin chromatography show that the complexity of the oligosaccharide chains are also altered during different phases of the human menstrual cycle and that less complex FSH isoforms 
are found during mid-cycle than in early follicular or late luteal periods (Anobile et al., 1998). The marked shift in FSH distribution profile favouring less acidic isoforms of FSH coincides with the timing of preovulatory follicular development.

\section{FSH isoforms may differ in their functional attributes}

Before attributing physiological significance to heterogeneity, it is essential to determine whether the various isoforms differ in their functionalities. To be biologically meaningful, changes in FSH heterogeneity need to be of sufficient magnitude to alter the net potency of the hormone or have various functions. Estimations of the biopotencies of FSH isoforms on the basis of FSH mass predict a 5-8-fold difference in radio-receptor and biopotency of purified human pituitary-derived FSH isoforms as well as a fourfold discrepancy between radio-receptor and in vitro biopotencies (Burgon et al., 1993). Considering that subtle increases in immunoreactive FSH can induce ovarian responses (Ben-Rafael et al., 1995), potency differences such as those reported by Burgon et al. (1993) and the magnitude of changes such as those seen during mid-cycle (Padmanabhan et al., 1988a) and puberty (Padmanabhan et al., 1992) have the potential to have meaningful biological consequences. In this context it is of interest that pulsatile administration of GnRH leads to fast clearing FSH signals in patients with idiopathic hypogonadotropic-hypogonadism (Padmanabhan et al., 1988b) and a less negatively charged (less acidic) form of FSH in children with pubertal disorders (Wide et al., 1996b). Similarly treatment with a GnRH antagonist leads to production of a basic form of FSH that is capable of antagonizing FSH action (Dahl et al., 1988).

Less acidic (pI 5.0-5.6) isoforms of human recombinant FSH have been shown to be more potent than those in the mid- ( $\mathrm{pI} 3.6-4.6$ ) and acidic ( $\mathrm{pI} 4.5-5.0$ ) ranges in inducing mouse follicular development in vitro (Vitt et al., 1997). In addition to inducing follicles of large final size, the less acidic FSH isoforms, even at the lowest concentration tested $\left(25 \mathrm{mIU} \mathrm{ml}^{-1}\right)$, induced antral formation in $70 \%$ of follicles and oestrogen production by day 2 of exposure to FSH. A similar degree of follicle development was achieved only with $100 \mathrm{mUU} \mathrm{ml} \mathrm{m}^{-1}$ of the mid- $\mathrm{pI}$ value and $500 \mathrm{mIU} \mathrm{ml} \mathrm{m}^{-1}$ of acidic FSH isoforms. Furthermore, oestradiol secretion was evident only after 4 days of culture even with the $500 \mathrm{mUU}_{\text {acidic }} \mathrm{FSH} \mathrm{ml}-1$. These studies indicate that the physicochemical nature of FSH isoforms may lead to quantitative and qualitative differences in ovarian function.

An exciting possibility is that the different FSH isoforms encode different functions. On the basis of the multitude of functions. FSH mediates at the gonads, involvement of different second messenger systems and potential for receptor cross-talk (Fig. 6), the signal transduction pathway of FSH isoforms may differ and culminate in altered responses. There is precedence with other glycoprotein hormones for such altered signal transduction cascades. FSH and thyroid-stimulating hormone (TSH) have been shown to bind each others receptors (Dobozy et al., 1985). Interaction of TSH with human FSH receptor is a possible mechanism by which some children with juvenile hypothyroidism exhibit unexplained precocious puberty (Anasti et al., 1995). Asialo human chorionic gonadotrophins (hCGs) have been shown to have higher TSH-like activity in human thyroid follicles (Yamazaki et al., 1995). In this context, it is of interest that there is a positive correlation between serum free thyroxine and asialo hCG in patients with gestational thyrotoxicosis (Tsuruta et al., 1995). Similarly, different isoforms of TSH have been shown to have different biological activities: the basic isoforms promoting iodide and thymidine uptake and acidic isoforms increasing intracellular cAMP (Pickles et al., 1992). Certain L.H isoform(s) have also been shown to possess renotrophic activity but weak steroidogenic potential (Nomura et al., 1988). Studies with deglycosylated and native mixes of ovine FSH also show isoform specific cAMP and oestradiol responses (Fig. 1) (Padmanabhan, 1995). These observations provide support for the possibility that endocrine-induced changes in FSH heterogeneity can have functional consequences.

\section{Conclusion}

Our understanding of mechanisms controlling the pubertal process and ovulatory cyclicity may be flawed, in as much as the basis for such concepts is derived mainly from immunological 


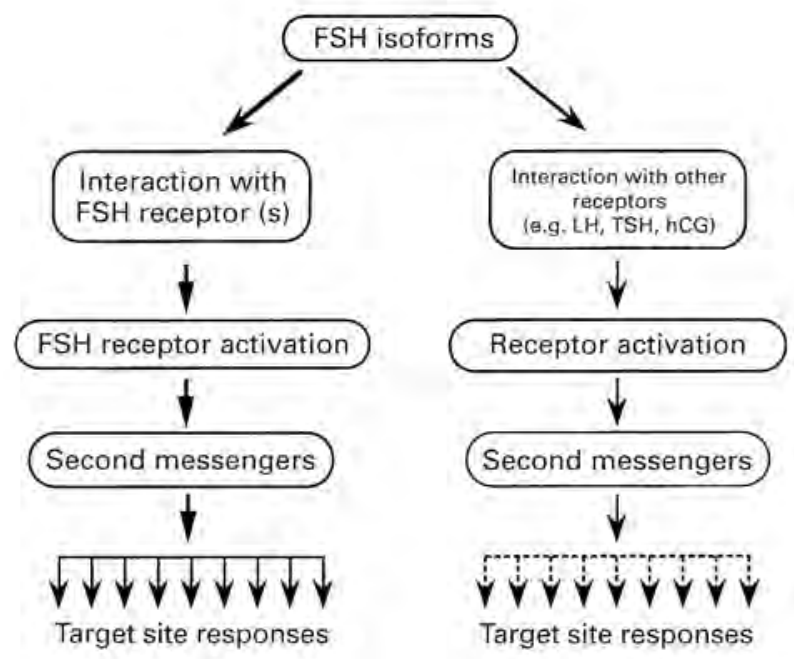

Fig. 6. Schematic diagram showing the potential sites at which the effector mechanisms may vary for the different FSH isoforms leading ultimately to differential target site responses. Different FSH isoforms may show differential affinity to a given FSH receptor, or selectively bind variant FSH receptor population or even structurally unrelated receptors (receptor cross-talk). Changes in receptor binding in turn can lead to changes in signalling cascades involving different second messenger systems and culminate in differential target cell responses.

characterizations of circulating patterns of gonadotrophic hormones that may not recognize the entire repertoire of gonadotrophin variants present in circulation. Mounting evidence indicates that, through an interaction with the hypothalamus and the gonad, the anterior pituitary gland can secrete different types of FSH that vary in biological potency and circulatory half-life. This opens up very interesting questions such as whether target cells differentially respond to the pattern of the imposed FSH signals, much as the T cell is known to differentially respond to small changes in ligand (Marx, 1995). In addition, the molecular modifications responsible for these observed changes in the distribution of FSH isoforms have the potential to lead to changes in affinity for classical FSH receptors, cross-reactivity with non-FSH receptors as well as with any other FSH receptor forms that might have been induced by the same endocrine conditions that led to the changes in FSH. From these perspectives, changes in FSH heterogeneity have the potential to provide an exquisitely finetuned mechanism to control gonadal function.

Therefore, it is a matter of great importance: (1) to determine details of the linkage between changes in isoform distribution and modifications in the biological attributes of the FSH signal delivered to the gonad during different physiological and pathological states; such efforts need to use well characterized assay systems and reference preparations that are reflective of the repertoire of FSH isoforms present in biological fluids, and (2) to reveal whether the various isoforms differentially act to initiate distinct functions in target cells. Only when the structure and importance of the various naturally occurring FSH isoforms are determined will it be possible for us to obtain a complete understanding of the mechanisms regulating reproductive processes. If heterogeneity proves to be biologically important, it should be possible to design FSH isoforms for desired functions and to use them too regulate fertility. 


\section{References}

Anasti JN, Flack MR, Froehlich J, Nelson LM and Nisula BC (1995) A potential novel mechanism for precocious puberty in juvenile hypothyroidism Journal of Clinical Endocrinology and Metabolisur $80276-279$

Anobile CJ, Talbot JA, McCann JA, Padmanabhan V and Robertson WR (1998) Glycoform composition of serum gonadotrophins through the normal menstrual cycle and in post-menopausal state Humar Reproduction 4 641-647

Beitins IZ and Padmanabhan V (1991) Bioactivity of gonadotropins Endocrinology and Metabolism Clinics of North America 20 85-120

Ben-Rafael Z, Levy T and Schoemaker J (1995) Phàrmacokinetics of follicle-stimulating hormone: clinical significance Fertility and Sterility 63 689-700

Blum WFP and Gupta D (1980) Age and sex-dependent nature of the polymorphic forms of rat pituitary FSH: the role of glycosylation Neuroendocrinology Letters 2 357-365

Burgon PG, Robertson DM, Stanton PG and Hearn MTW (1993) Immunological activities of highly purified isoforms of human FSH correlate with in vitro bioactivities Journal of Endocrinology 139 511-518

Chappel SC (1995) Heterogeneity of follicle stimulating hormone: control and physiological function Human Reproduction Update 1 479-487

Chappel SC, Ulloa-Aguirre A and Coutifaris C (1983) Biosynthesis and secretion of follicle-stimulating hormone Endocrine Reviews 4 179-211

Christine-Maitre S, Taylor AE, Khoury RH, Hall JE, Martin KA, Smith PC, Albanese C, Jameson JL, Crowley WE, Jr and Sluss PM (1996) Homologous in vifro bioassay for folliclestimulating hormone (FSH) reveals increased FSH biological signal during the mid-phase to late-luteal phase of the human menstrual cycle Journal of Clinical Endocrinology and Metabolism $812080-2088$

Cooke DJ, Crowe MA and Roche JF (1997) Circulating FSH isoform patterns during recurrent increases in FSH throughout the oestrous cycle of heifers lournal of Reproduction and Fertility 110 339-345

Crowe MA, Padmanabhan V, Mihm M, Beitins IZ and Roche JF (1998) Resumption of follicular waves in beef cows is not associated with peri-parturient changes in FSH heterogeneity despite major changes in steroid and gonadotropin concentrations Biology of Reproduction $\mathbf{5 8}$ $1445-1450$

Dahl KD, Bicsak TA and Hsueh AJW (1988) Naturally occurring antihormones: secretion of FSH antagonists by women treated with a GnRH analog Science 239 72-74

Damián-Matsumura P, Zaga V, Sánchez-Hernández C, Maldonado A, Timossi C and Ulloa-Aguire A (1998) The changes in $\alpha$ 2,3 , sialyltransferase mRNA levels during the rat estrous cycle and after castration correlate with variations in charge distribution of intrapituitary follicle-stimulating hormone (FSH) Program and Abstracts of the 80th Annual Meeting of the Endoctine Society, New Orleans p90 (Abstract OR 28-6)

Dharmesh SM and Baenziger JU (1993) Estrogen modulates expression of the glycosyltransferases that synthesize sulfated oligosaccharides on lutropin Proceedings of the National Academy of Sciences USA 90 11127-11131

Ding Y-Q, Ranta T, Nikkanen I and Huhtaniemi I (1991) Discordant levels of serum bioactive $\mathrm{LH}$ in man as measured in different in-vitro bioassay systems using rat and mouse interstitial cells and human granulosa-luteal cells Joumal of Endoctinology 128 131-137

Dobozy O, Csaba G, Hetenyi G and Shahin M (1985) Investigation of gonadotropin-thyrotropin overlapping and hormonal imprinting in the rat testis Acta Physiologica Hungarica $66169-175$

Flores JA, Veldhuis JD and Leong DA (1990) Follicle-stimulating hormone evokes an increase in intracellular free calcium ion concentrations in single ovarian (granulosa) cells Endocrinology 127 3172-3179

Gudermann T, Brockman H, Simoni M, Gromoll J and Nieschlag E (1994) In vitro bioassay for serum follicle-stimulating hormone (FSH) based on L. cells transfected with recombinant rat $\mathrm{FSH}$ receptor: validation of a model systèm Endocrinology 135 2204-2213

Harsch IA, Simoni M and Nieschlag E (1993) Molecular heterogeneity of serum follicle-stimulating hormone in hypogonadal patients before and during androgen replacement therapy and in normal men Clinical Endocrinology 39 173-180

Hassing JM, Kletter GB, I'Anson H, Woods RI, Beitins IZ, Foster DL and Padmanabhan V (1993) Pulsatile administration of gonadotropin-releasing hormone does not alter the follicle-stimulating hormone (ESH) isoform distribution pattern of pituitary or circulating FSH in nutritionally growth-restricted ovariectomized lambs Endocrinology 132 $1527-1536$

Jaakkola T, Ding YQ, Kellokumpu-Lehtinen P, Valavaara R, Martikainen H, Tapanainen J, Ronnberg L and Huhtaniemi I (1990) The ratios of serum bioactive/immunoreactive luteinizing hormone and follicle-stimulating hormone in various clinical conditions with increased and decreased gonadotropin secretion: reevaluation by a highly sensitive immunometric assay Journal of Clinical Endocrinology and Metabolism 70 1496-1505

Jia XC, Kessel B, Yen SSC, Tucker EM and Hsueh AJW (1986) Serum bioactive follicle-stimulating hormone during the human menstrual cycle and in hyper- and hypogonadotrophic states: application of a sensitive granulosa cell atomatase bioassay Joumal of Clinical Endocrinology and Metabolism 62 1243-1249

Lee JS, Manning JM, Foster DL and Padmanabhan V (1998) Estrogen increases the secretion of less-acidic FSH isoforms in ovariectomized prepubertal and peripubertal lambs Program and Abstracts of the 80ti. Anmual Meeting of the Endocrine Society, New Orlears p. 348 (Abstract P2-474)

Marx J (1995) The T cell receptor begins to reveal its many facets Science $267459-460$

Morell AG, Gregoriadis G and Scheinberg IH (1971) The role of sialic acid in determining the survival of glycoproteins in circulation Journal of Biological Chemistry 246 1461-1467

Nomura K, Tsunasawa S, Ohmura K, Sakiyama F and Shizume K (1988) Renotropic activity in ovine luteinizing hormone isoform(s) Endocrinology 123 700-712

Padmanabhan V (1995) Neuroendocrine control and physiologic relevance of FSH heterogeneity Journal of Reproduction and Fertility Abstract Series 15 Abstract S3

Padmanabhan V, Chappel SC and Beitins IZ (1987) An improved in vitro bioassay for follicle-stimulating hormone (FSH): suitable for measurement of FSH in unextracted human serum Endocrinology 121 1089-1098

Padmanabhan V, Lang LL, Sonstein I, Kelch RP and Beitins 1 Z 
(1988a) Modulation of serum follicle-stimulating hormone bioactivity and isoform distribution by estrogenic steroids in normal women and in gonadal dysgenesis Journal of Clinical Endocrinology and Meiabolism 67 465-473

Padmanabhan V, Kelch RP, Sonstein J, Foster CM and Beitins IZ (1988b) Bioactive follicle-stimulating hormone responses to intravenous gonadotropin-releasing hormone in boys with idiopathic hypogonadotropic hypogonadism fournal of Clinical Endocrinology and Metabolism 67 793-800

Padmanabhan V, Mieher CD, Borondy M, I'Anson H, Wood RI, Landefeld TD, Foster DL and Beitins IZ (1992) Circulating bioactive follicle-stimulating hormone and less acidic follicle-stimulating hormone isoforms increase during experimental induction of puberty in the female lamb Endocrinology 131 213-220

Padmanabhan V, McFadden K, Mauger DT, Karsch FJ and Midgley AR, Jr (1997a) Neuroendocrine control of folliclestimulating hormone (FSH) secretion: I. Direct evidence for separate episodic and basal components of FSH secretion Endocrinology 138424432

Padmanabhan V, I'anson $\mathrm{H}_{\ell}$ Foster DL and Beitins IZ (1997b) Estradiol increases the release of less acidic FSH isoforms in nutritionally growth-retarded lambs Program and Abstracts of the 79th Annual Meeting of the Endocrine Society Abstract P3-336

Pettersson KSI and Soderhölm JR-M (1991) Individual differences in lutropin immunoreactivity revealed by monoclonal antibodies Clinical Chemistry 37 333-340

Phillips DJ and Wide L (1994) Serum gonadotropin isoforms become more basic after an exogenous challenge of gonadotropin-releasing hormone in children undergoing pubertal development Joumal of Clinical Endocrinology and Metabolism $79814-819$

Pickles AJ, Peers N, Robertson WR and Lambert A (1992) Different isoforms of human pituitary thyroid-stimulating hormone have different relative biological activities Journal of Molecular Endocrinology 9 251-256

Robertson DM, Foulds LM, Fry RC, Cummins JT and Clarke I (1991) Circulating half-lives of follicle-stimulating hormone and luteinizing hormone in pituitary extracts and isoform fractions of ovariectomized and intact ewes Endocrinology 129 1805-1813

Simoni M, Khan SA and Nieschlag E (1991) Serum bioactive follicle-stimulating hormone-like activity in human pregnancy is a methodological artifact Journal of Clinical Endocrinology and Metabolism 73 1118-1122

Simoni M, Jockenhövel F and Nieschlag E (1993a) Biological and immunological properties of the international standard for FSH 83/575: isoelectrofocusing profile and comparison with other FSH preparations Acta Endocrinologica 128 281-288

Simoni M, Weinbauer GF and Nieschlag E (1993b) Molecular composition of two different batches of urofollitropin: analysis by immunofluorimetric assay, radioligand receptor assay and in vitro bioassay Joumal of Endocrinological Invesfigations 1621-27

Simoni M, Jockenhövel F and Nieschlag E (1994) Polymorphism of human pituitary FSH: analysis of immunoreactivity and in vitro bioactivity of different molecular species Joumal of Endocrinology 141 359-367

Simoni M, Peters J, Behre HM, Kliesch S, Leifke E and Nieschlag E (1996) Effects of gonadotropin-releasing hormone on bioactivity of follicle-stimulating hormone (FSH) and microstructure of $\mathrm{FSH}$, luteinizing hormone and sex- hormone binding globulin in a testosterone-based contraceptive trial: evaluation of responders and nonresponders European Journal of Endocrinology $135433-439$

Spiegel AM, Shenker AM and Weinslein LS (1992) Receptor-effector coupling by $\mathrm{G}$ proteins: implications for normal and abnormal signal transduction Endocrive Reviews 13: 536-565

Stanton PG, Burgon PG, Hearn MTW and Robertson DM (1996) Structural and functional characterization of hFSH and $\mathrm{hLH}$ isoforms. Molecular and Cellular Endocrinology 125 133-141

Stumpf TT, Roberson MS, Wolfe MW, Zalesky DD, Cupp AS, Werth LA, Kojima N, Hejl K, Kittok RJ, Grotjan HE and Kinder JE (1992) A similar distribution of gonadotropin isohormones is maintained in the pituitary throughout sexual maturation in the heifer Biology of Reproduction 46 $442-450$

Timossi CM, de Tomasi JB, Zambrano E, González R and UlloaAguirre A (1998) A naturally occurring basically charged human follicle-stimulating hormone (FSH) variant inhibits FSH-induced androgen aromatization and tissue-type plasminogen activator enzyme activity in vilro. Neuroendocrinology 67 153-163

Tsuruta E, Tada H, Tamaki H, Kashiwai T, Asahi K, Takeoka K, Mitsuda N and Amino N (1995) Pathogenic role of asialo human chorionic gonadotropin in gestational thyrotoxicosis Toumal of Clinicat Endocrinology and Metabolism 80 350-355

Ulloa-Aguirre A, Midgley AR, Jr, Beitins IZ and Padmanabhan V (1995) Follicle-stimulating isohormones: characterization and physiological relevance Endocrine Reviews 16 765-787

Vitt UA, Kloosterbocr H], Rose UM, Kiesel PS, Bete A and Nayudu PL (1997) Differential effects of 3 hFSH isoforms on ovarian follicle development in vitro foumal of Reproduction and Fertility Abstract Series 20 Abstract 22

Wide $\mathrm{L}$ and Bakos O (1993) More basic forms of both human follicle-stimulating hormone and luteinizing hormone in serum at midcycle compared with the follicular and luteal phase Journal of Clinical Endocrinology and Metabolism 76 885-889

Wide $\mathrm{L}$ and Naessern T (1994) 17ß-Oestradiol counteracts the formation of the more acidic isoforms of folliclestimulating hormone and luteinizing hormone after menopause Clinical Endocrinology (Oxford) 40 783-789

Wide L, Naessén T, Eriksson K and Rune C (1996a) Time-related effects of progestogen on the isoforms of serum gonadotrophins in $17 \beta$-oestradiol treated postmenopausal women Clinical Endocrinology 44 651-658

Wide L, Albertsson-wikland K and Phillips DJ (1996b) More basic isoforms of serum gonadotropins during gonadotropinreleasing hormone therapy in pubertal children Journal of Clinical Endocrinology and Metabolism 81 216-221

Yamazaki K, Sato K, Shizume K, Kanaji Y, Ito Y, Obara T, Nakagava T, Koizumi T and Nishimura R (1995) Potent thyrotropic activity of human chorionic gonadotropin variants in terms of 125 incorporation and de novo synthesized thyroid hormone release in human thyroid follicles journal of Clinical Endocrinology and Metabolism 80 473-479

Zambrano E, Olivares A, Mendez JP, Guerrero L, Diaz-Cueto L, Veldhuis JD and Ulloa-Aguirre A (1995) Dynamics of basal and gonadotropin-releasing hormone-releasable serum follicle stimulating hormone charge isoform distribution throughout the human menstrual cycle Journal of Clinical Endocrinology and Metabolism 80 1647-1656 